\title{
Pharmacotherapy of inflammatory process by ginger extract (Zingiber officinale) ointment
}

\author{
Iryna Kravchenko ${ }^{1,2}{ }^{\circledR}$, Lidiia Eberle $^{1^{*} \mathbb{\oplus}}$, Mariia Nesterkina $^{1,2}{ }^{\mathbb{C}}$, Alona Kobernik $^{1}$ \\ ${ }^{1}$ Department of Pharmaceutical Chemistry, I.I. Mechnikov Odessa National University, Odessa, Ukraine \\ ${ }^{2}$ Department of Organic and Pharmaceutical Technology of Odessa National Polytechnic University, Odessa, Ukraine
}

\section{A R T I C L E IN F O}

Article Type:

Original Article

\section{Article History:}

Received: 17 May 2018

Accepted: 24 January 2019

Keywords:

Carrageenan inflammation

Ginger extract

Seromucoids

Sialic acids

Total protein

Protein fractions

\begin{abstract}
A B S T R A C T
Introduction: The anti-inflammatory activity of the ointment based on ginger extract was investigated on carrageenan-induced inflammation.

Methods: Anti-inflammatory activity was investigated on the carrageenan inflammation model in rat. The animals were divided into 5 groups: first group- control (without treatment); second group- animals received preventive treatment of ointment with ginger extract two days before experiment and with subsequent treatment after administration of phlogogen; 3rd group- animals received applications of 5\% ibuprofen 2 days before experiment and with subsequent treatment after induction of inflammation; fourth group - animals were treated with ginger extract ointment after one day of the phlogogen administration; fifth group animals were treated with reference drug after one day of the phlogogen introduction. The biochemical parameters (concentration of sialic acids, seromucoids, contents of total proteins and protein fractions) of the blood of experimental animals were investigated.

Results: After local application of the ointment from the ginger extract, under the conditions of its application after phlogogen inoculation, the effect was the same as anti-inflammatory effect of the ibuprofen which was used as reference drug. The preventive ointment application provided faster and more intense reduction of the inflammatory focus with returning to the background indicators on the eighth day of the experiment, and for biochemical parameters on the sixth day of therapy.

Conclusion: The ointment from ginger extract shows anti-inflammatory activity by inhibiting inflammation caused by carrageenan.
\end{abstract}

Implication for health policy/practice/research/medical education:

Zingiber officinale extract possesses significant anti-inflammatory and analgesic action and, consequently, might be used as an effective natural agent for pain and inflammation after transdermal delivery.

Please cite this paper as: Kravchenko I, Eberle L, Nesterkina M, Kobernik A. Pharmacotherapy of inflammatory process by ginger extract (Zingiber officinale) ointment. J Herbmed Pharmacol. 2019;8(2):101-107. doi: 10.15171/jhp.2019.16.

\section{Introduction}

The anti-inflammatory medicines play a leading role in treating many inflammatory diseases in clinical practice. Despite the large number of anti-inflammatory medicines, the problem of effectiveness and safety of the inflammation therapy has not been resolved, yet. The searching of the effective anti-inflammatory medicines, which would have no side effect while maintaining a powerful anti-inflammatory effect, remains a pressing challenge in modern pharmacology. Phytotherapy is one of the promising directions for the construction of safe and effective anti-inflammatory medicines $(1,2)$. In recent years, interest in herbal medicines has grown. Their advantages over synthetic medicines are mild physiological action, minimal toxicity, the possibility of prolonged use, the action without addiction and immunosuppression. Ginger root is one of the medicinal plants used for many years in the traditional medicine in the treatment of inflammatory diseases of the joints (3-5).

According to the literature the value of ginger as a medicinal plant, provide polyphenolic compounds, such as 6-gingerol ( 48.2\%), 8-gingerol and 10-gingerghalaglic acid $(\sim 21.5 \%)$, chlorogenic acid $(\sim 14.2 \%)$ and caffeic acid $(\sim 14.2 \%)$. 6-Gingerol is the most important compound 
from the big variety of compounds with different chain length from the gingerol family.

It was found that 6-gingerol suppresses cyclooxygenase (COX), lipoxygenase (LOX) and synthesis of prostaglandins (PG); blocks transient receptors potential cation channel subfamily V member 1 (TRPV1); suppresses intracellular synthesis of heat shock proteins (HSPs), as well as substances that inhibit collagen synthesis in the extracellular matrix (ECM); blocks serotonin receptors; increases oxygen consumption by cells (1-5).

In addition, ginger contains $\beta$-carotene, capsaicin, caffeine acid and curcumin. Ginger contains a full range of essential amino acids (tryptophan, threonine, leucine, methionine, phenylalanine, valine), a complex of vitamins, magnesium, calcium and phosphorus salts (4).

Therefore, the aim of this study was to investigate the anti-inflammatory activity of ointment made from the ginger extract on the model of carrageenan-induced inflammation with determination of the preferred mechanism of action.

\section{Materials and Methods}

A viscid extract from Zingiber officinale was obtained by extraction on a ultrasonic disintegrator (ultrasonic disintegrator type UD-11) of shredded root with $70 \%$ ethanol in the ratio of raw material:solvent - 1:1. After 2 weeks of additional maceration, the solvent was distilled off under vacuum to form a viscid extract (5).

Anti-inflammatory activity was investigated on the carrageenan inflammation model, which was induced by subplantar injection to the plantar fasciitis (aponeurosis) of the hind limb of rats using 0.1 mLof $1 \%$ carrageenan (6).

For treatment, $0.025 \%$ ointment from viscid ginger extract was used, which, according to the previous studies had the most pronounced anti-exudative effect on short inflammation models (7). Ibuprofen (5\%) was used as a reference medicine.

The experiment was performed on 50 male Wistar rats (with approximate weight 200-220 g), which in turn were divided into 5 groups of 10 animals in each:

First group - control (animals without treatment);

Second group - animals received preventive treatment of ointment with ginger extract 2 days before the start of the experiment and with subsequent treatment after administration of phlogogen;

Third group - animals received applications of 5\% ibuprofen 2 days before the start of the experiment and with subsequent treatment after induction of inflammation;

Fourth group - animals were treated with ginger extract ointment after 1 day of the phlogogen administration;

Fifth group - animals were treated with reference medicine after 1 day of the phlogogen introduction.

The dynamic of changes of the inflammatory process was evaluated by measuring the volume of the affected limb (by volumetric method) daily for 8 days. The ointment with the $Z$. officinale extract and the reference medicine was applied to the affected limb once a day, and the obtained results were compared to the output in the percentage.

All studies conformed to the rules of the "European Convention for the Protection of Vertebrate Animals used for Experimental and Other Scientific Purposes" (8) and the principles of the Ukrainian National Congress on Bioethics (9).

The ointment was tested to see the impact on the development and the course of anti-exudative activity was assessed by its ability to reduce the swelling of the limbs, the content of sialic acids, total protein, and the ratio of protein fractions in the blood serum of experimental animals. Biochemical studies were performed prior to the study and on first, third, sixth and eighth days of the experiment. Biochemical parameters such as content of sialic acids, total protein and protein fractions were determined by the commercial test kits for rapid analysis manufactured by Research and Production Enterprise "Filicit-Diagnostika" Ltd. (Ukraine).

Statistical significance was calculated using one-way ANOVA followed by Tukey's multiple comparison test. $P$ $<0.05$ was considered as significant.

\section{Results}

The acute inflammatory process caused by the inoculation of carrageenan during the first day of the experiment in all groups of animals (Figure 1) led to the development of an inflammatory reaction with the paw volume increase (an average of $80 \%$ ), edema (swelling) and limitation of the movement of the hind limbs. However, the peak volume of the inflammatory focus for the control group was observed on the third day that exceeded intact rates by 2 times. Application of ointment with ibuprofen and ointment with ginger extract reduced the volume of affected limbs after the first treatment (after 24 hours), both in the groups of animals with the preventive use of ointments and groups treated after the introduction of phlogogen (for all groups $P<0.05$ was considered as significant).

In groups of animals, which had preventively application of ointment ( 2 days) before the experiment, the increase in the volume of the inflammatory focus was not differ from the control group on the first day of the experiment. However, the next application of ointments after 1 day suppressed volume growth and blocked the further development of acute exudative inflammation after administration of the phlogogenic agent compared to the control group. The application of both ibuprofen and ointment with ginger extract contributed to a decrease in the volume of affected limbs after the first time of the treatment, next day it was observed a tendency to decrease the volume of affected limbs.

At the end of the experiment (eighth day), the volume of the affected limbs of the experimental groups (second 

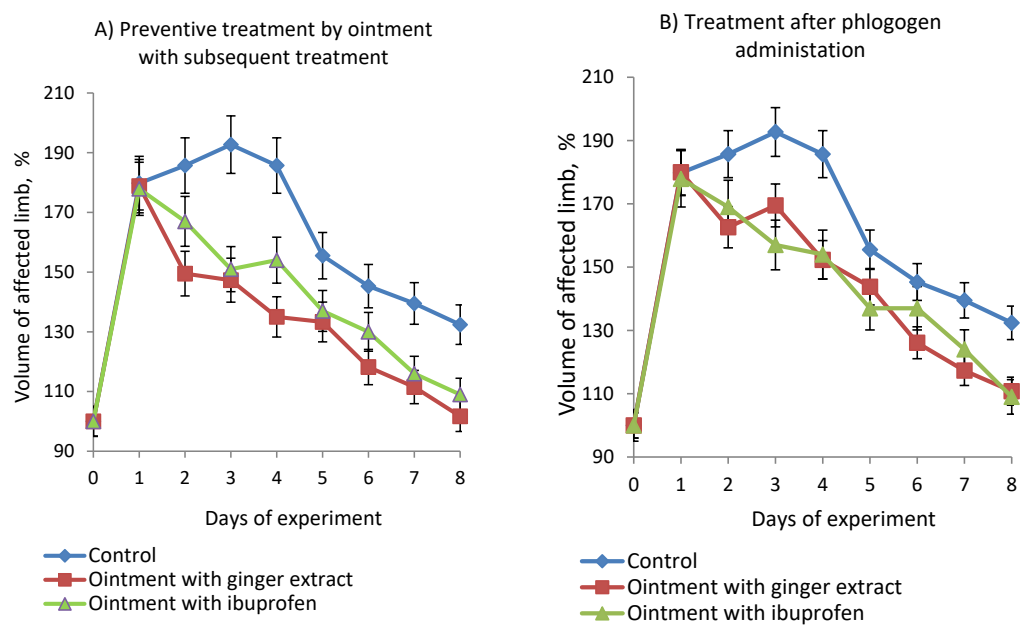

Figure 1. Anti-inflammatory activity of experimental ointments under different conditions of the ointment application. A) Preventive treatment by ointment with subsequent treatment, B) treatment after phlogogen administration.

and third group) returned to initial value, while the control group exceeded initial value by about $40 \%$. At the same time, it was observed not only the disappearance of morphological characteristics of inflammation such as edema, redness, but also the restoration of functional activity. Starting from the eighth day of treatment, the rats were able to stand on their affected limbs, palpation was painless, and mobility in the joints was completely restored.

On third day after the induction of carrageenan inflammation, the maximum increase in concentrations of sialic acids in the blood of experimental animals was observed (approximately in 1.2-1.5 times) compared to initial value.

On the sixth and eighth days of the experiment, the content of sialic acid in the serum of experimental rats gradually started to decrease. In the third and fifth animal groups (treated by ibuprofen ointment) a significant decrease in sialic acid content on the sixth and eighth days of therapy was observed; this indicator has almost reached the level of intact animals (Table 1).

In the case of using an ointment based on ginger extract, this indicator dropped faster and more intensively, under the conditions of preventive application of the experimental ointment (second group), the level of sialic acids reached the initial values almost on sixth day of the experiment and was $2.23 \pm 0.13 \mathrm{mMol} / \mathrm{L}$, whereas, in the fourth group treated after the administration of the phlogogen, the decrease was observed on the eighth day of the study $-2.42 \pm 0.16 \mathrm{mMol} / \mathrm{L}$, which slightly exceeded the initial values. In the control group of animals which were not treated, the level of sialic acids was outside the range of physiological norm and it was $2.84 \pm 0.17 \mathrm{mMol} / \mathrm{L}$ on the eighth day.

The next stage of the study was to determine changes in the dynamics of seromucoids in serum, which, along with sialic acids, is an important prognostic indicator for connective tissue damage and the presence of inflammatory reactions in the body. According to our data, an increase in seromucoids content after inflammation induction during the first and third days was raised by 2.5-3 times relative to initial values (Table 2 ).

Therapeutic measures using ointment in conditions of its preventive application (prior to the administration of phlogogen) led to reduction of seromucoids level in serum on the eighth day of the experiment $(0.97 \pm 0.06$ turbidity units). Additionally, in animal group that had ointment application after phlogogen administration on the

Table 1. Content of sialic acid in blood serum of rats ( $\mathrm{mMol} / \mathrm{L})$

\begin{tabular}{lcccc}
\hline \multirow{2}{*}{$\begin{array}{l}\text { Days of the experiment } \\
\text { Control }\end{array}$} & \begin{tabular}{c} 
Preventive application of the ointment \\
\cline { 3 - 5 } extract
\end{tabular} & $\begin{array}{c}\text { Treatment after the phlogogen inoculation } \\
\text { ibuprofen }\end{array}$ & $\begin{array}{c}\text { Ointment with ginger } \\
\text { extract }\end{array}$ & $\begin{array}{c}\text { Ointment with } \\
\text { ibuprofen }\end{array}$ \\
\hline Before phlogogen administration & $2.24 \pm 0.12$ & $2.17 \pm 0.09$ & $2.26 \pm 0.08$ & $2.11 \pm 0.15$ \\
1 & $2.65 \pm 0.18$ & $2.47 \pm 0.11$ & $2.51 \pm 0.13$ & $2.64 \pm 0.12$ \\
3 & $3.78 \pm 0.14$ & $2.51 \pm 0.15^{*}$ & $3.27 \pm 0.16$ & $3.29 \pm 0.19$ \\
6 & $3.12 \pm 0.23$ & $2.23 \pm 0.13^{*}$ & $2.81 \pm 0.11$ & $3.46 \pm 0.11$ \\
8 & $2.84 \pm 0.17$ & $2.16 \pm 0.12^{*}$ & $2.53 \pm 0.07$ & $2.96 \pm 0.24$ \\
\hline
\end{tabular}

$* P<0.05$ compared to control group 
Table 2. Content of seromucoids in blood serum of rats (turbidity units)

\begin{tabular}{|c|c|c|c|c|c|}
\hline \multirow[b]{2}{*}{ Days of the experiment } & \multirow[b]{2}{*}{ Control } & \multicolumn{2}{|c|}{ Preventive application of the ointment } & \multicolumn{2}{|c|}{ Treatment after the phlogogen inoculation } \\
\hline & & $\begin{array}{l}\text { Ointment with ginger } \\
\text { extract }\end{array}$ & $\begin{array}{l}\text { Ointment with } \\
\text { ibuprofen }\end{array}$ & $\begin{array}{l}\text { Ointment with ginger } \\
\text { extract }\end{array}$ & $\begin{array}{l}\text { Ointment with } \\
\text { ibuprofen }\end{array}$ \\
\hline Before phlogogen administration & $0.98 \pm 0.07$ & $0.93 \pm 0.09$ & $0.95 \pm 0.07$ & $0.94 \pm 0.08$ & $0.97 \pm 0.06$ \\
\hline 1 & $1.46 \pm 0.11$ & $1.28 \pm 0.13$ & $1.45 \pm 0.12$ & $1.36 \pm 0.12$ & $1.49 \pm 0.16$ \\
\hline 3 & $2.73 \pm 0.15$ & $1.86 \pm 0.17 *$ & $2.17 \pm 0.18$ & $2.14 \pm 0.19$ & $2,12 \pm 0.25$ \\
\hline 6 & $2.11 \pm 0.17$ & $1.31 \pm 0.12 *$ & $1.53 \pm 0.17^{*}$ & $1.62 \pm 0.15^{*}$ & $1.43 \pm 0.17^{*}$ \\
\hline 8 & $1.86 \pm 0.12$ & $0.97 \pm 0.06 *$ & $1.02 \pm 0.13^{*}$ & $1.07 \pm 0.14 *$ & $0.98 \pm 0.11 *$ \\
\hline
\end{tabular}

$* P<0.05$ compared to control group

eighth day of experiment, seromucoids level was slightly increased and equalled to $1.07 \pm 0.14$ turbidity units, which indicates the beginning of reparative processes of connective tissue.

The animals from the control group had the content of seromucoids remained at an average of $1.86 \pm 0.12$ turbidity units on the eighth day that surpasses the initial numbers more than in 2 times. The reduction in the content of seromucoids had already observed on the sixth day of the experiment and on the eighth day it almost has reached the initial numbers in third and fifth animal groups using ibuprofen.

The total plasma protein is the total concentration of all circulating proteins and it is a main component of the blood. According to the literature (10), the decrease in this indicator is observed in endogenous intoxication (11), enhanced protein catabolism or its redistribution due to the exit of proteins from the bloodstream with the formation of transudative-exudative complexes in the acute inflammation (12).

In the study of the content of total protein in blood plasma of rats, after 1 day of inflammation induction, results showed a significant decrease in the concentration of total protein (hypoproteinaemia) almost in 2 times in all experimental groups of animals compared with the initial numbers (Figure 2). According to literary sources, hypoproteinaemia in the conditions of carrageenan inflammation is associated with the release of proteins from the vascular channel into the inflammatory focus that accumulate outside the vessels leading to the formation and accumulation of the exudate. Whereas the degradation of the protein molecules into smaller ones and oncotic pressure increasing in the tissues, which, in turn, leads to redness and swelling of the limb in the inflammatory foci (6).

Further therapeutically usage in experimental animal groups stimulated a gradual increase in total protein, but in some groups, on the sixth and eighth day of the experiment, the return of protein content to background numbers was noticed.

In the analysis of total proteins in the blood of animals from the second group (under the conditions of preventive application of the ointment based on the ginger extract) on the third day of the experiment, a tendency was observed to increase the experimental index and on the sixth day it has already noted its return to the initial numbers $(76 \mathrm{~g} / \mathrm{L})$, whereas the restoration of the initial protein content was fixed on the eighth day of the study ( $72 \mathrm{~g} / \mathrm{L})$ was observed
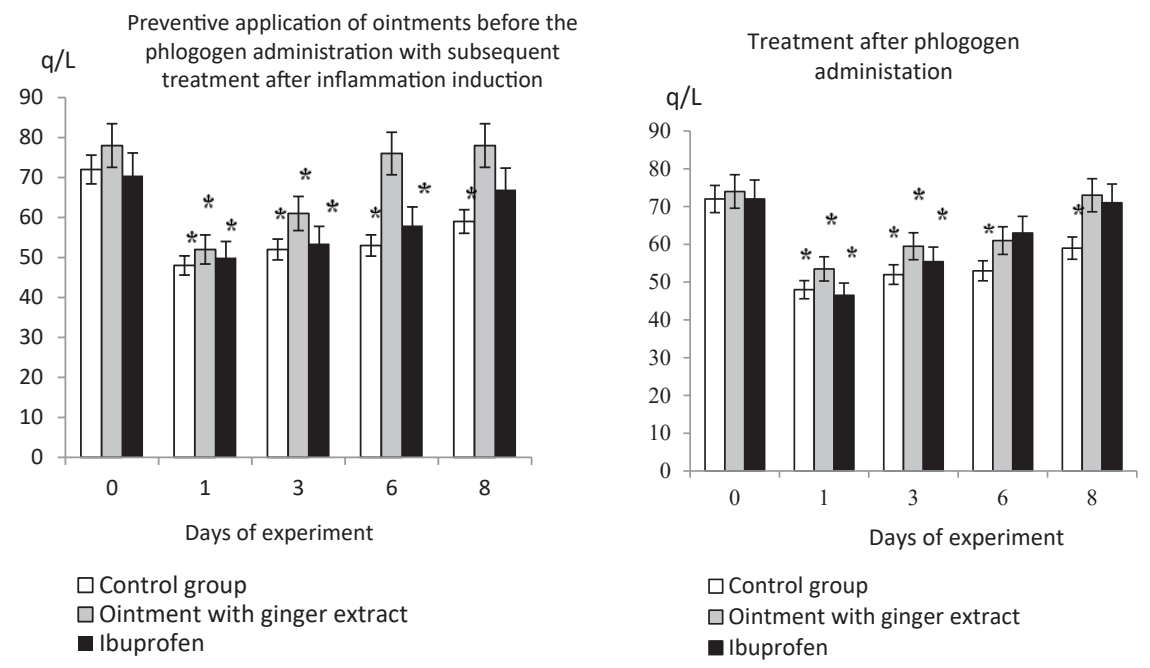

Figure 2. The content of total protein in blood serum of rats under different conditions of the ointment application 
in the fourth group (Figure 2).

In other experimental groups, the return of total protein to background numbers was also noted on the eighth day of the investigation, except for the control group, where the total protein count was lower throughout the investigation but exceeded the initial numbers at the end of the experiment.

Investigating the quantitative ratio between different serum proteins (albumin-globulin ratio) together with the total protein helps to more accurately identifying specific changes during the applied therapy and allows to determine which fraction lead to increasing of proteins or their deficiency.

According to literature (13), albumins are the most important by a quantitative ratio, since they determine $80 \%$ of the information about the colloid-osmotic pressure on the blood plasma, directly involved in providing buffer capacity, increase the reserves of free amino acids in the body, etc.

One day after phlogogen inoculation, there were changes in the albumin-globulin ratio, including the decrease in albumin concentration (hypoalbuminemia) in all animal groups in the range of $21 \%-38 \%$, while the level of some globulins, the so-called proteins of acute phase, increased in 2 times in comparison with physiological norm indicators. Hypoalbuminemia, according to literature data, is associated with an increase in the level of interleukin-1 (IL-1), the acute phase reaction of inflammation, and the immunological process activation with increasing of the immunoglobulins formation (14), that leads to a decrease in the albumin content in the blood in 1.9-2.4 times.

Investigation of the protein fractions in experimental animal groups showed that the smallest changes in the albumin-globulin ratio were observed in second group under the conditions of the previous application of the ointment based on ginger extract. The phlogogen administration in this group of animals did not cause a significant increase in the volume of acute phase proteins (a1-globulins for 18\%, a2-globulins - 29\%, $\gamma$-globulins - 48\%). Increasing such of proteins in the second group were the same as in other experimental groups, and further treatment stimulated the restoration of the albumin-globulin ratio on the sixth day of the experiment (Figure 3).

The IL-1 action on hepatocytes leads to a decrease in the synthesis of albumins and an increase in the production of "acute phase" proteins (15). An integral part of the biological effect of IL-1 is its stimulating effect on the metabolism of connective tissue. It stimulates the fibroblasts proliferation and increases the PG production, growth factors and a number of cytokines. The connective tissue cells increase the synthesis of collagen, collagenase, and other enzymes under IL-1 influence.

According to the total protein analysis and the protein fractions parameters in the second animal group, ointment based on ginger extract was able to restore the protein metabolism in the blood on the sixth day of the investigation, whereas ointments application in third, fourth, fifth animal groups, did not show such a therapeutic effect, and the protein fractions coefficients were increased by 1.5-2 times compared to initial rates.

The protein fractions concentration was increased throughout the experiment and on the eighth day it was outside the background in the control group.

Based on the above, it is possible to conclude that ointment based on ginger extract had a pronounced antiexudative activity compared to the control group and it was the same as with the reference medicine results in terms of the treatment after the inflammation induction.

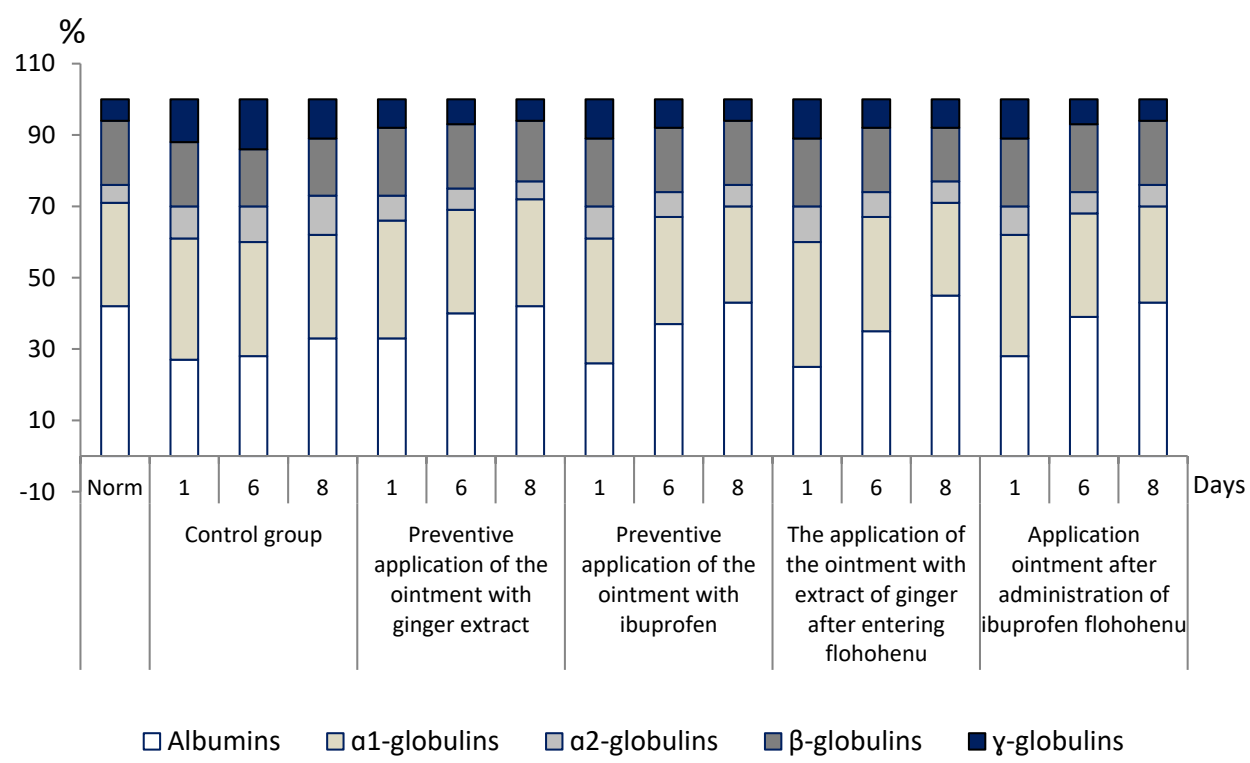

Figure 3. The ratio of protein fractions (\%) on first, sixth and eighth day after inflammation induction under different conditions of ointment application. 
The preventive ointment application around 2 days before the inflammation injection was effective and superior to the reference medicine in accordance with both morphological and biochemical researches.

\section{Discussion}

The valuable pharmacologically active substances contained in the root of $Z$. officinale make this raw material a promising agent for the treatment of inflammatory and pain conditions. This is probably due to their ability to suppress the activity of COX, an enzyme that regulates the biotransformation of arachidonic acid to prostaglandins (PG), especially prostaglandin I2 (prostacyclin or PGI2) and thromboxane.

Since COX-2 is one of the important integrators of inflammatory stimuli, it might be considered as a promising target in the treatment. The anti-exudative effect of the ginger extract is realized by inhibiting the activity of COX, by reducing the biological synthesis of eicosanoids - metabolites of unsaturated fatty acids that are involved in the pathogenesis of inflammation. It should be noted that, the therapeutic effect of ginger extract is realized by inhibiting the activity of COX-2 without affecting COX-1 inhibition in contrast to non-steroidal anti-inflammatory medicine (6).

The performed research showed the effectiveness of an ointment based on ginger extract as an anti-inflammatory agent in the case of carrageenan-induced inflammation. Preventive ointment application can increase the amount of extract that has penetrated through the skin, thereby increasing its concentration in the inflammatory focus, resulting the increased therapeutic efficacy. Evidence of the effectiveness of preventive ointment application was the external indicators of the inflammatory process reduction and the normalization of biochemical parameters in experimental groups compared with the group of animals, where the ointment was applied after the phlogogen introduction.

The biochemical parameters of serum are also of indisputable diagnostic value, along with the morphological characteristics of the inflammatory process. As inflammatory markers, we selected the following indicators: sialic acids, seromucoids, total protein content and protein fraction ratio. According to the literature data, the maximum increase in the concentration of seromucoids on the third day of the experiment is due to the connective tissue destruction in the acute phase of the inflammatory process, which is followed by a massive penetration of the seromucoids in the blood plasma.

Sialic acids, that are $\mathrm{N}$ - and O-acyl derivatives of neuraminic acid, are considered as structural components of glycolipids of biomembranes, glycoproteins and proteoglycans of connective tissue and biological fluids. In free form, they are present in blood, mucous membrane of the stomach, thyroid gland, and usually can be found in the composition of mucous membranes of saliva, and glycoproteins of the skin $(16,17)$. That is why the detection of sialic acids in biological fluids is widely used as a diagnostic marker. Increasing the concentration of sialic acids in blood serum of rats in conditions of acute exudative inflammation, according to literature sources, is associated with a deviation of tissue metabolism and depolymerisation of glycoprotein complexes, therefore it is possible to detect products of decomposition of proteincarbohydrate complexes that appear in serum.

The protein metabolism investigation showed that reducing the concentration of seromucoids and sialic acids, increasing the total protein and protein fractions in serum during treatment of inflammation with ginger extract, indicate destructive and reparative processes in the inflammatory focus, which are manifested by a general decrease in systemic inflammatory response compared with the control group.

\section{Conclusion}

The ginger extract application as a local antiinflammatory and anesthetic agent significantly reduced the morphological and biochemical manifestations of the inflammatory process by probably blocking the COX pathway of metabolism of arachidonic acid, which in turn led to a decrease in the synthesis of PGs and PGI2, inhibited the release of inflammatory mediators from eosinophils. Using the obtained results from the antiexudative activity of ginger extract from the root on the model of carrageenan inflammation, further research is promising for its detailed study on other inflammation models in order to find out the possible mechanisms of anti-inflammatory activity of the extract and preparation of new drugs.

\section{Authors' contributions}

IK conceived and designed the experiment; LE and AK performed the experiments; IK, LE and AK analyzed the data; IK, LE, AK and MN wrote the paper. All authors read and confirmed publication of the paper.

\section{Conflict of interests}

The authors declare no conflict of interest.

\section{Ethical considerations}

Ethical issues including plagiarism, misconduct, data fabrication, falsification, double publication or submission have been carefully checked by authors.

All experimental procedures conform to the guiding principles for research as recommended by "Guide for the Care and Use of Laboratory Animals" (NIH publication 86-23 revised 1985).

\section{Funding/Support}

None. 


\section{References}

1. Bhat SV, Nagasampagi BA, Sivakumar M. Chemistry of natural products. New Delhi, India: Narosa Publishing House; 2005:326.

2. Shukla Y, Singh M. Cancer preventive properties of ginger: a brief review. Food Chem Toxicol. 2007;45(5):683-90.

3. Dugasani SB, Pichika MR, Nadarajah VD. Comparative antioxidant and anti-inflammatory effects of [6]-gingerol, [8]-gingerol, [10]-gingerol and [6]-shogaol. J Ethnopharmacology. 2009;127:515-520.

4. Kokate CK, Purohit AP, Gokhale SB. Pharmacognosy. Pune: Nirali publication; 2009:107-128.

5. Kravchenko I, Kobernik A, Eberle L. Optimization of extraction methods for total polyphenolic compounds obtained from rhizomes of Zingiber officinale. Trends Phytochem Res. 2018;2(1):37-42.

6. Stefanov OV. Preclinical studies of drugs. Kiev: Avicena; 2001:528.

7. Kravchenko I, Kobernik A, Eberle L. Anti-inflammatory activity of solid ginger extract (Zingiber officinale) under transdermal introduction. J Pharmacol Drug Toxicol. 2017;6(56):43-49.

8. European convention for the protection of vertebrate animals used for experimental and other scientific purposes. Strasbourg: Council of Europe; 1986: 1-51.

9. Swiss Academy of Medical Sciences (SAMS) and Swiss
Academy of Sciences (SCNAT). Ethical Principles and Guidelines for Experiments on Animals. 3rd ed. London. 2005.

10. Eghtesad S, Poustchi H, Malekzadeh R. Malnutrition in liver cirrhosis: The influence of protein and sodium. Middle East J Dig Dis. 2013; 5(2):65-75.

11. Fairbanks KD, Tavill MD, Tavill AS. Liver disease in alpha 1-antitrypsin deficiency: A review. Am J Gastroenterol. 2008;103:2136-2141.

12. Dideriksen K, Reitelseder S, Holm L. Influence of amino acids, dietary protein, and physical activity on muscle mass development in humans. Nutrients. 2013;5:852-876.

13. Finfer S, Bellomo R, Boyce N, et al. A comparison of albumin and saline for fluid resuscitation, in the intensive care unit. N Engl J Med. 2004;22:2247-56. doi: 10.1056/ NEJMoa040232.

14. Quinlan GJ, Martin GS, Evans TW. Albumin: biochemical properties and therapeutic potential. Hepatology. 2005;41:1211-1219. doi: 10.1002/hep.20720

15. Fournier T, Medjoubi N, Porquet D. Alpha-1acid glycoprotein. Biochim Biophys Acta. 2000;1482:157-71.

16. Kamyshnikov VS. List manual clinical and biochemical laboratory diagnosis. Minsk: Belarus; 2000:64-66.

17. Dolgov VV, Shevchenko OP. Laboratory diagnosis of disorders of protein metabolism [in Russian]. Nauka. $2003 ; 67$. 